\title{
Percentage* of Adults Aged $\geq 18$ Years Who Did Not Take Their Medication as Prescribed or Asked for Lower-Cost Medication to Save Money Among Those Prescribed Medication in the Past 12 Months, ${ }^{\dagger}$ by Number of Chronic Conditions $^{\S}$ - National Health Interview Survey, 2018
}

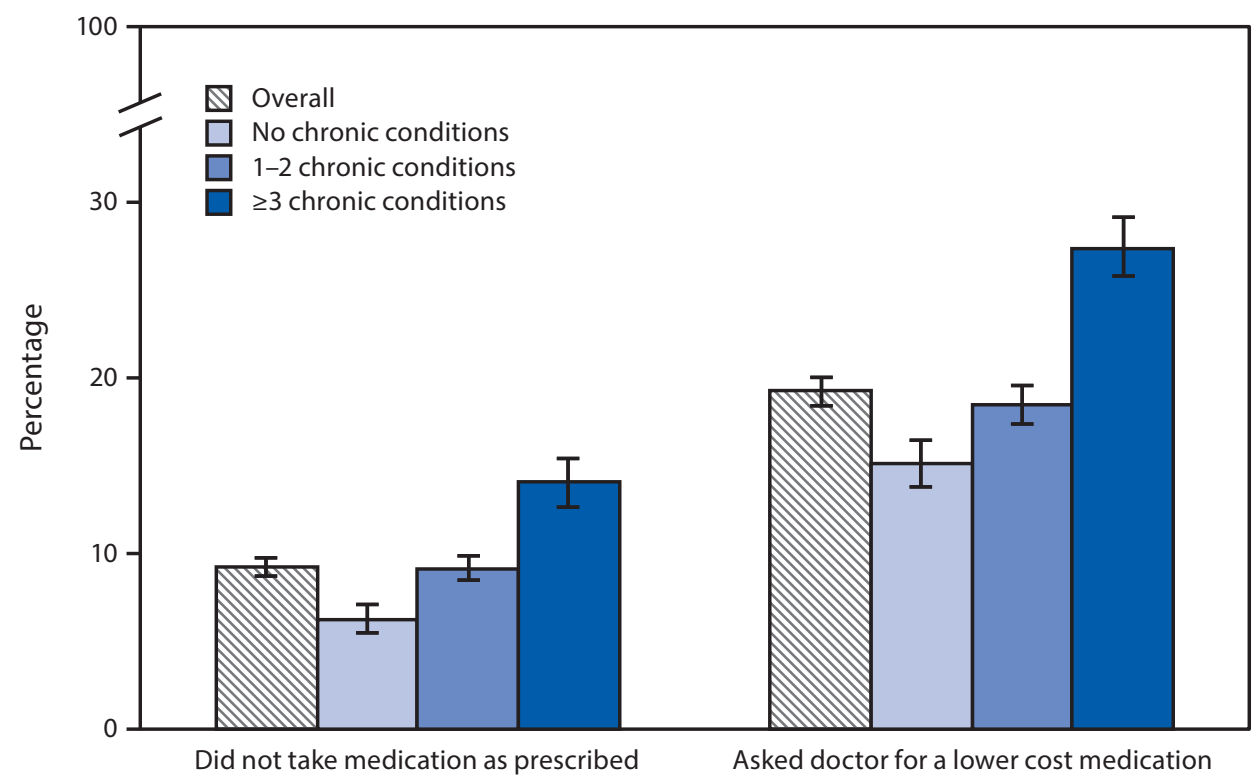

* With 95\% confidence intervals indicated by error bars.

† Based on the following questions asked of adults prescribed medication in the past 12 months: During the past 12 months, were any of the following true for you? 1) You skipped medication doses to save money, 2) You took less medication to save money, 3) You delayed filling a prescription to save money, or 4) You asked your doctor for a lower-cost medication to save money. The category "Did not take medication as prescribed" includes adults who skipped medication doses, took less medication, or delayed filling a prescription.

$\S$ The number of chronic conditions is based on reporting ever being diagnosed with: hypertension, coronary heart disease, stroke, diabetes, cancer, arthritis, hepatitis, chronic obstructive pulmonary disease (COPD) or asthma, or reporting weak or failing kidneys in the past 12 months. COPD was defined as ever having COPD or emphysema or having chronic bronchitis during the past 12 months.

I Estimates are based on household interviews of a sample of the civilian, noninstitutionalized, U.S. population and are derived from the National Health Interview Survey Sample Adult component.

In 2018, among adults aged $\geq 18$ years who were prescribed medication in the past 12 months, the percentage who did not take their medication as prescribed to save money increased with the number of reported chronic conditions, from $6.2 \%$ with no chronic conditions to $9.1 \%$ with $1-2$ chronic conditions and $14.0 \%$ with $\geq 3$ chronic conditions. The percentage who asked their doctor for a lower-cost medication also increased with the number of reported chronic conditions from $15.1 \%$ among those with no chronic conditions to $18.4 \%$ among those with $1-2$ chronic conditions and $27.4 \%$ among those with $\geq 3$ chronic conditions.

Source: National Health Interview Survey, 2018 data. https://www.cdc.gov/nchs/nhis.htm.

Reported by: Amy E. Cha, PhD, oty6@cdc.gov, 301-458-4236; Robin A. Cohen, PhD. 